PROCEEDINGS OF THE

AMERICAN MATHEMATICAL SOCIETY

Volume 130, Number 7 , Pages 1993-1996

S 0002-9939(01)06278-5

Article electronically published on December 20, 2001

\title{
A SEMINORM WITH SQUARE PROPERTY ON A COMPLEX ASSOCIATIVE ALGEBRA IS SUBMULTIPLICATIVE
}

\author{
ZOLTÁN SEBESTYÉN \\ (Communicated by N. Tomczak-Jaegermann) \\ Dedicated to Professor Franek Szafraniec on the occasion of his sixtieth birthday

\begin{abstract}
The result stated in the title is proved as a consequence of an appropriate generalization replacing the square property of a seminorm with a similar weaker property which implies an equivalence to the supnorm of all continuous functions on a compact Hausdorff space also.
\end{abstract}

Theorem. Let $p$ be a seminorm with the square property on a complex (associative) algebra $A$. Then the following hold for all $a, b$ in $A$ :

(1) $p(a b-b a)=0$.

(2) $p(a b) \leq p(a) p(b)$.

This is a unifom seminorm analogue of [8] or Thm. 6 in [6] that a $\mathbb{C}^{*}$-seminorm is submultiplicative (and the involution is isometric). We answer a problem posed in [3] and solved in the particular case of Banach algebras [4].

A seminorm on $A$ is a nonnegative function on $A$ satisfying:

(i) $p(a+b) \leq p(a)+p(b)$ for all $a, b$ in $A$ and

(ii) $p(\lambda a)=|\lambda| p(a)$ for all $a$, for all scalars $\lambda$.

The seminorm $p$ is submultiplicative if

(iii) $p(a b) \leq p(a) p(b)$ for all $a, b$ in $A$.

It satisfies the square property [3, 4] if

(iv) $p\left(a^{2}\right)=p(a)^{2}$ for all $a$ in $A$.

The above theorem is a consequence of the following.

Proposition. Let $p$ be a seminorm on a complex (associative) algebra A satisfying (iv) $)^{*} m(a)^{2} \leq p\left(a^{2}\right) \leq M p(a)^{2}$ for all $a$ in $A$, where $0<m \leq M$ are given constants. Then properties (1) and $(2)^{*}$,

$(2)^{*} m p(a b) \leq M^{2} p(a) p(b)$ for all $a, b$ in $A$,

hold true.

Received by the editors September 6, 2000 and, in revised form, January 17, 2001.

2000 Mathematics Subject Classification. Primary 46H05, 46 J05.

Key words and phrases. Seminorm with the square property on an algebra, submultiplicative seminorm.

(C)2001 American Mathematical Society 
Proof of the Proposition. We argue step by step as follows.

First step. $p(a b+b a) \leq 4 M p(a) p(b)$ for all $a, b$ in $A$.

This is nothing more than Lemma 2.1 in [1. We include the proof for the sake of completeness. The identity

$$
2(a b+b a)=(a+b)^{2}-(a-b)^{2}
$$

implies that

$$
\begin{aligned}
2 p(a b+b a) & \leq p\left((a+b)^{2}\right)+p\left((a-b)^{2}\right) \leq M p(a+b)^{2}+M p(a-b)^{2} \\
& \leq 2 M(p(a)+p(b))^{2}
\end{aligned}
$$

and so

$$
p(a b+b a) \leq 4 M \text { for all } a, b \text { in } A \text { with } p(a) \leq 1, p(b) \leq 1 .
$$

Now, for any $\varepsilon>0$ we find that

$$
p\left(\frac{a}{p(a)+\varepsilon}\right)<1, \quad p\left(\frac{b}{p(b)+\varepsilon}\right)<1,
$$

hence that

$$
p(a b+b a) \leq 4 M(p(a)+\varepsilon)(p(b)+\varepsilon) .
$$

So the first step is done.

Second step. $p(b a b) \leq 10 M^{2} p(a) p(b)^{2}$ for all $a, b$ in $A$.

The identity

$$
2 b a b=(a b+b a) b-a b^{2}+b(a b+b a)-b^{2} a
$$

enables us to conclude by the first step that

$$
\begin{aligned}
2 p(b a b) & \leq p((a b+b a) b+b(a b+b a))+p\left(a b^{2}+b^{2} a\right) \\
& \leq 4 M p(a b+b a) p(b)+4 M p(a) p\left(b^{2}\right) \\
& \leq 16 M^{2} p(a) p(b)^{2}+4 M^{2} p(a) p(b)^{2},
\end{aligned}
$$

and the second step follows.

Third step. $m p(a b-b a)^{2} \leq 96 M^{3} p(a)^{2} p(b)^{2}$ for all $a, b$ in $A$.

The identity

$$
(a b-b a)^{2}=2[a(b a b)+(b a b) a]-(a b+b a)^{2}
$$

implies by the first two steps that

$$
\begin{aligned}
m p(a b-b a)^{2} & \leq p\left((a b-b a)^{2}\right) \\
& \leq 2 p(a(b a b)+(b a b) a)+p\left((a b+b a)^{2}\right) \\
& \leq 8 M p(a) p(b a b)+M p(a b+b a)^{2} \\
& \leq 80 M^{3} p(a)^{2} p(b)^{2}+16 M^{3} p(a)^{2} p(b)^{2} .
\end{aligned}
$$

Fourth step. $p(a b) \leq(2+5 \sqrt{M / m}) M p(a) p(b)$ for all $a, b$ in $A$.

This is an easy consequence of the former steps:

$$
\begin{aligned}
2 p(a b) & =p((a b+b a)+(a b-b a)) \leq p(a b+b a)+p(a b-b a) \\
& \leq 4 M p(a) p(b)+10 M \sqrt{\frac{M}{m}} p(a) p(b),
\end{aligned}
$$

since $96 \leq 10^{2}$ and the step is done. 
Fifth (final) step.

According to the fourth step the kernel of $p, \operatorname{ker} p$, is a two-sided ideal in the algebra $A$; therefore the norm $|\cdot|$ on the quotient algebra $A / \operatorname{ker} p$, defined by

$$
|a+\operatorname{ker} p|:=p(a) \text { for all } a \text { in } A,
$$

satisfies property (iv)*, hence the consequences stated in the first four steps. Define an algebra norm

$$
\|a+\operatorname{ker} p\|:=\sup \{|\lambda a+a b+\operatorname{ker} p|:|\lambda|+|b+\operatorname{ker} p| \leq 1 ; \lambda \in \mathbb{C}, b \in A\}
$$

for all $a$ in $A$

on $A / \operatorname{ker} p$ as in 1.1.9 Prop. in [7]. We find that

$$
|a+\operatorname{ker} p| \leq\|a+\operatorname{ker} p\| \leq\left(2+5 \sqrt{\frac{M}{m}}\right) M|a+\operatorname{ker} p|
$$

and that $\|\cdot\|$ satisfies property (iii), i.e. it is submultiplicative.

We note that property (iv)* holds also as follows:

$$
m^{2^{n}-1} p(a)^{2^{n}} \leq p\left(a^{2^{n}}\right) \leq M^{2^{n}-1} p(a)^{2^{n}} \text { for all } a \text { in } A \text { and } n=1,2, \ldots
$$

The norm $|\cdot|$ on $A / \operatorname{ker} p$ thus also fulfils

$$
m^{2^{n}-1}|a+\operatorname{ker} p|^{2^{n}} \leq\left|a^{2^{n}}+\operatorname{ker} p\right| \leq M^{2^{n}-1}|a+\operatorname{ker} p|^{2^{n}} .
$$

We conclude that the spectral radius $r$ in the normed algebra $A / \operatorname{ker} p$ satisfies

(v) $\|a+\operatorname{ker} p\| \leq\left(2+5 \sqrt{\frac{M}{m}}\right) \frac{M}{m} r(a+\operatorname{ker} p)$ for all $a$ in $A$.

Indeed, we find that with $C=\left(2+5 \sqrt{\frac{M}{m}}\right) M$

$$
\begin{aligned}
\|a+\operatorname{ker} p\| & \leq C|a+\operatorname{ker} p| \leq \frac{C}{m^{1-2^{-n}}}\left|a^{2^{n}}+\operatorname{ker} p\right|^{2^{-n}} \\
& \leq \frac{C}{m^{1-2^{-n}}}\left\|a^{2^{n}}+\operatorname{ker} p\right\|^{2^{-n}}, \quad n=1,2, \ldots,
\end{aligned}
$$

and the statement follows as $r(a+\operatorname{ker} p)=\lim _{n \rightarrow \infty}\left\|a^{2^{n}}+\operatorname{ker} p\right\|^{2^{-n}}$. The HirschfeldZelazko Theorem (see 3.1.7 Prop. in [7], (B.6.17) Cor. in [5] or [2, Lemma 2, p. 46]) now gives by (v) that $A / \operatorname{ker} p$ is commutative, i.e. property (1) holds.

Finally property $(2)^{*}$ follows in consequence of the following argument: since

$$
\begin{aligned}
m^{2^{n}-1} p(a b)^{2^{n}} & \leq p\left((a b)^{2^{n}}\right)=\left|(a b)^{2^{n}}+\operatorname{ker} p\right| \leq\left\|(a b)^{2^{n}}+\operatorname{ker} p\right\| \\
& =\left\|a^{2^{n}} b^{2^{n}}+\operatorname{ker} p\right\| \leq\left\|a^{2^{n}}+\operatorname{ker} p\right\|\left\|b^{2^{n}}+\operatorname{ker} p\right\| \\
& \leq C^{2}\left|a^{2^{n}}+\operatorname{ker} p \| b^{2^{n}}+\operatorname{ker} p\right|=C^{2} p\left(a^{2^{n}}\right) p\left(b^{2^{n}}\right) \\
& \leq C^{2} M^{2^{n+1}-2} p(a)^{2^{n}} p(b)^{2^{n}}
\end{aligned}
$$

holds for $n=1,2, \ldots$ we see that property $(2)^{*}$ holds true indeed.

We conclude with an affirmative answer to a question in [4, Remarks (5)].

Corollary. Let $A=C(K)$ be the Banach algebra with supnorm $\|\cdot\|_{\infty}$ of all continuous functions on a compact Hausdorff space $K$. Let $|\cdot|$ be a norm on $C(K)$ with property (iv)*. Then $|\cdot|$ is equivalent to $\|\cdot\|_{\infty}$. 
Proof. Noting that $\|\cdot\|_{\infty}$ is the spectral radius in $C(K)$ we have by the proof of the Proposition that with the algebra norm $\|\cdot\|$ above we have at once that

$$
\frac{m}{C}|a| \leq \frac{m}{C}\|a\| \leq\|a\|_{\infty} \leq\|a\| \leq C|a| \text { for all } a \text { in } A .
$$

Here we use the fact that $\|\cdot\|_{\infty} \leq\|\cdot\|$ automatically holds true (see 2.4.15 Theorem in [7]).

Note added (December, 2000). In the square property (iv), the inequality " $<$ or $="$ would be more natural for the conclusion (iii). However, this is not true: the numerical radius for Hilbert space operators, e.g. for 2-by-2 matrices, fulfils property (iv) or even the power inequality $p\left(a^{n}\right) \leq p(a)^{n}$ and is not submultiplicative (see Theorem 3.1 in [1]).

It is also a natural question of whether the submultiplicativity of a seminorm (with the square property) implies subadditivity. A two-dimensional counterexample follows: $\mathbb{C}$ as a two-point function algebra has multiplicative and nonsubadditive seminorm of the form $p((w, z))=\sqrt{|w z|}$ since, e.g. $p((1,3))+p((3,1))=\sqrt{3}+\sqrt{3}<$ $4=p((4,4))$.

The author is indebted to the referee for calling attention to some misprints and questions above.

\section{ACKNOWLEDGMENT}

The author is highly indebted to J. Kristóf for doing the third step in the proof of the Proposition and also thanks the Széchenyi Professorship for their kind support.

\section{REFERENCES}

[1] R.Arens, M.Goldberg and W.A.J.Luxenburg, Multiplicative Factors for Seminorms II, J.Math.Anal.Appl. 170(1992), 401-413. MR 93k:46039

[2] B.Aupetit, Propriétés Spectrales des Algèbres de Banach, Lecture Notes in Math., vol. 735, Springer-Verlag, Berlin, Heidelberg and New York, 1979. MR 81i:46055

[3] S.J.Bhatt and D.J.Karia, Uniqueness of the uniform norm with an application to topological algebras, Proc.Amer.Math.Soc. 116(1992), 499-504. MR 92m:46068

[4] S.J.Bhatt, A seminorm with square property on a Banach algebra is submultiplicative, Proc.Amer.Math.Soc. 117(1993), 435-438. MR 93d:46072

[5] R.S.Doran and V.A.Belfi, Characterizations of $\mathbb{C}^{*}$-algebras: The Gelfand-Naimark Theorems, Pure and Applied Math. vol. 101, Marcel Decker, Inc., New York and Basel, 1986. MR 87k:46115

[6] Z.Magyar and Z.Sebestyén, On the definition of $\mathbb{C}^{*}$-algebras II, Can.J.Math. 37(1985), 664681. MR 87b:46061

[7] T.W.Palmer, Banach Algebras and General Theory of *-Algebras, Vol. I, Cambridge Univ. Press, 1994. MR 95c: 46002

[8] Z.Sebestyén, Every $\mathbb{C}^{*}$-seminorm is automatically submultiplicative, Period.Math.Hungar. 10(1979), 1-8. MR 80c:46065

Department of Applied Analysis, Loránd Eötvös University, H-1053 Budapest, KecsKEMÉTI U. 10-12, HUNGARY

E-mail address: sebesty@cs.elte.hu 